\title{
ANGULAR SIZE AND POSITION OF THE X-RAY SOURCE CYG-X-1
}

\author{
M. MATSUOKA, S. MIYAMOTO, J. NISHIMURA, M. ODA, Y. OGAWARA \\ Institute of Space and Aeronautical Science, University of Tokyo, Tokyo \\ and \\ M. WADA \\ Institute of Physical and Chemical Research, Tokyo
}

We performed a couple of balloon experiments to measure the size and the location of Cyg-X-1 using the techniques of the modulation collimator [1]. The angular periods of the modulation collimator were $26^{\prime}$ and $10^{\prime}$ for the respective flights corresponding to the approximate angular resolutions of the size determination and location, $3^{\prime}$ and $1^{\prime}$ respectively. Preliminary results of the experiment with $3^{\prime}$ resolution are reported here.*

The balloon was launched at Haranomachi, Japan at 0616 UT, September 23, 1968 and reached the ceiling of $8 \mathrm{~g} / \mathrm{cm}^{2}$ at $0820 \mathrm{UT}$. The passage of Cyg-X-1 at the meridian was expected at 1037 UT. The X-ray detector consists of two NaI(T1) square scintillators, each $3 \mathrm{~mm}$ thick and $85 \mathrm{~cm}^{2}$ area. The detector set underneath the modulation collimator detects the modulation of the X-ray flux while the X-ray source moves in the field of view and the orientation of the balloon gondola changes. We may determine the location of the source by means of the phase of the modulation and the size by the depth of the modulation.

A star sensor rigidly fixed to the structure which holds the modulation collimator was used to determine the aspect of the collimator with respect to the celestial sphere. The gondola was roughly pointed in such a way that one or two stars of Ursa Major

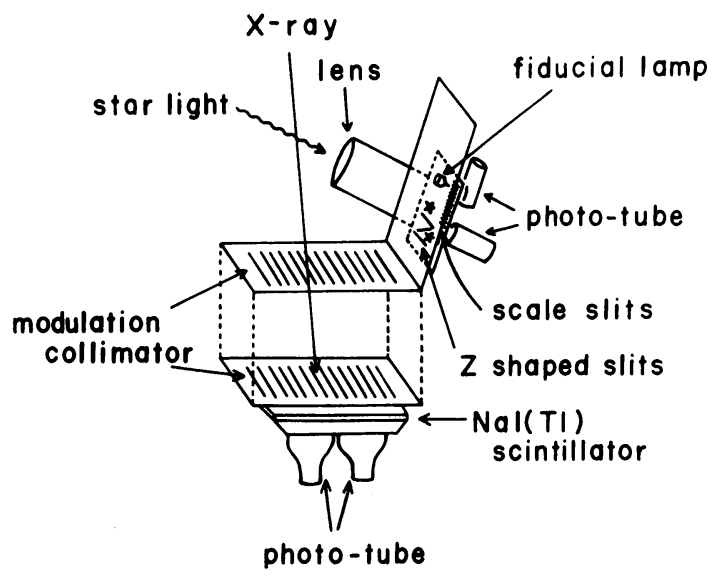

Fig. 1. Schematical figure of the balloon-borne modulation collimator and star sensor.

* The details of the instrument and data analysis will be published separately. 


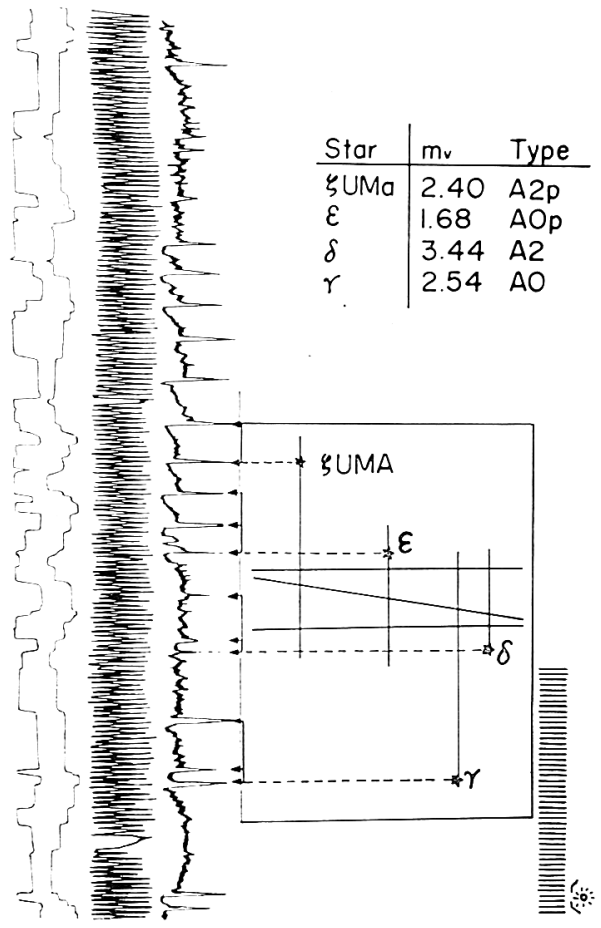

Fig. 2. Telemeter record showing the output pulses of star sensor and X-ray counters. The method to derive the direction of a star from the output pulses is illustrated.

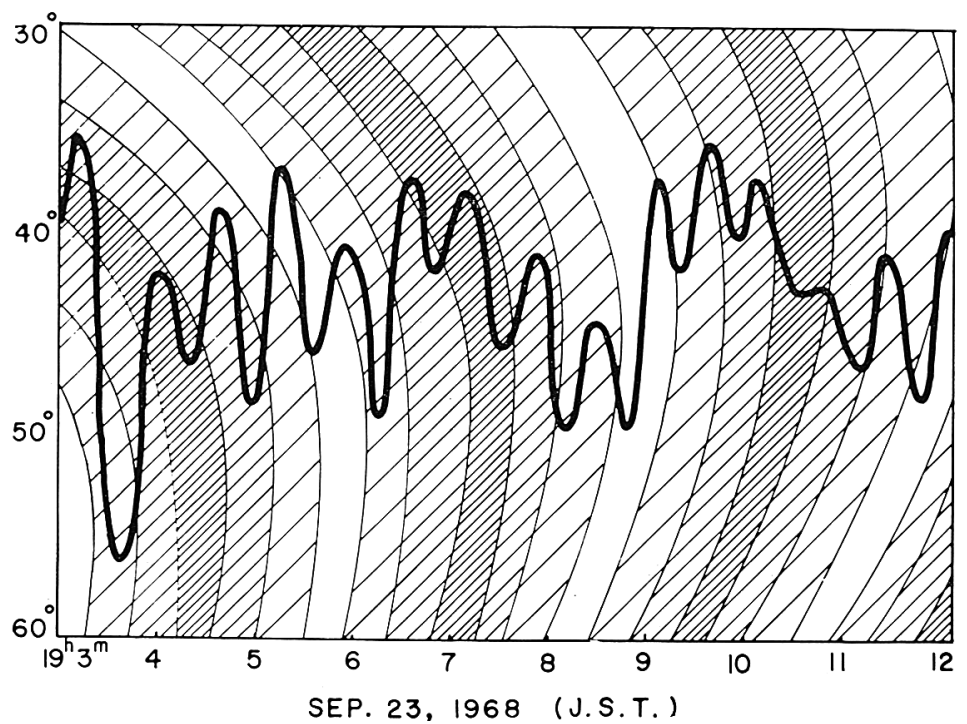

Fig. 3. The variation of the orientation of the instrument is obtained by means of the star sensor. The phase of expected modulation for a certain assumed position of an X-ray source is indicated. 
are always in the star sensor field of view. The star sensor is composed of a telephotolens and a moving diaphragm with slits at the focal plane of the lens which sweep star images and a fiducial lampmark. The Z-shaped slits cut on the diaphragm produce three light pulses for each star image. A row of slits on the diaphragm generates fiducial light pulses. Light pulses are converted to electric pulses by means of a photo multiplier and telemetered to the ground station.

An example of telemeter records is shown in Figure 2. The elevation and the azimuth of a star in the instrument frame of reference are obtained by the phase of appearance of star pulse with respect to the fiducial marks and the ratio of intervals between three pulses respectively. The precision of aspect determination using this star sensor has been proved to be less than an arc minute. The aspect was measured once every second.

A part of analysed data showing the variation of the orientation of the gondola is shown in Figure 3. If we assume a certain location of the source, we may draw contours as shown in the figure for phases of the transmission of the modulation collimator. Thus, we may synchronize X-ray counts and inspect the assumed location of the source for the modulation.

Data for one hour of observation have been analysed. A number of assumed points on the celestial sphere were thus tested for the phase and the amplitude of modulation. Figure 4 shows the candidate area of Cyg-X-1 in which good fits of data to the modulation obtained. The boundary of the area corresponds to one standard deviation error of the phase.

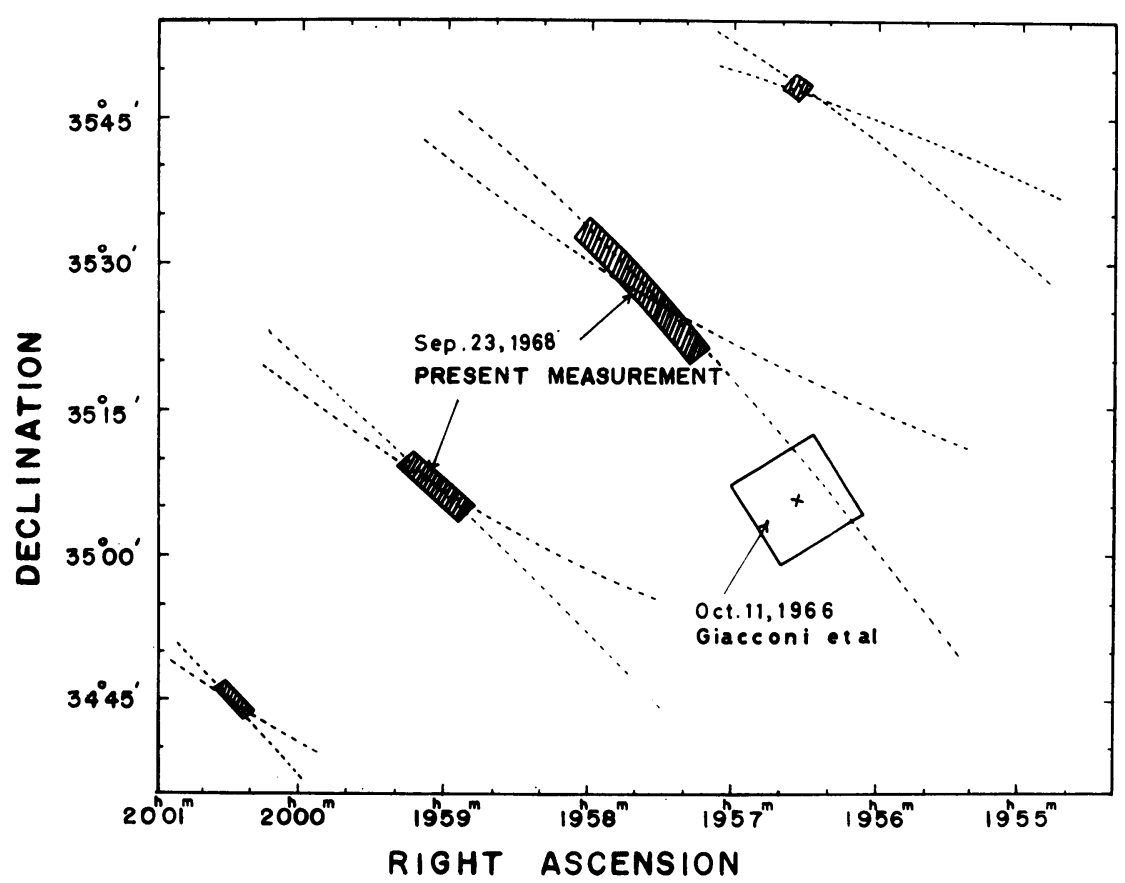

Fig. 4. Location of Cyg-X-1. (1950). 
Typical modulation is seen in Figure 5. The amplitude is consistent with the average observed intensity of Cyg-X-1, $0.14 \pm 0.01 / \mathrm{cm}^{2} \sec (25 \sim 50 \mathrm{keV})$. From this modulation curve we conclude that the depth of modulation is not reduced more than $10 \%$ of the phase. It is, thus, concluded that the upper limit of the size is three arc minutes.*

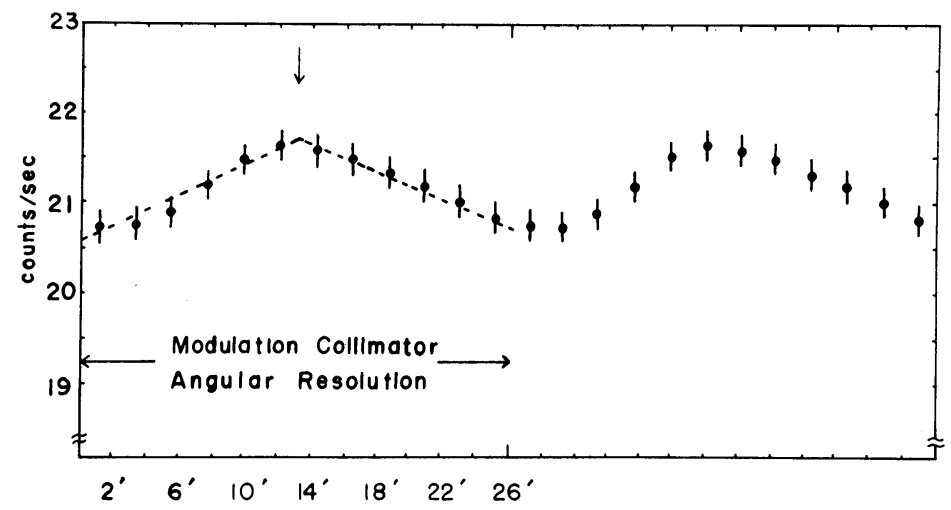

Fig. 5. The observed modulation of X-ray flux presented for two cycles.

The location shown in Figure 4 is in disagreement with the previously determined location by the ASE group [2] beyond the statistical error. We do not yet understand the cause of this disagreement and we feel we should reserve this part of our conclusions until further analysis is completed of the fine measurement which is more free from the systematic error in the relative angle of the optical axis of the star sensor and the collimator. This reservation does not affect the present conclusion on the size of the source.

\section{Acknowledgement}

We wish to acknowledge valuable assistance by Mr. M. Fujii and Mr. S. Ohta.

\section{References}

[1] Bradt, H., Garmire, G., Oda, M., Spada, G., and Sreekantan, B. V.: 1968, Space Sci. Rev. 8, 471.

[2] Giacconi, R., Gorenstein, P., Gursky, H., and Waters, J. R.: 1967, Astrophys. J. 148, L119.

* F. Floyd concluded that the upper limit was one arc minute, also utilizing a modulation collimator flown on a balloon (1969, Nature 222, 967). 